\title{
Studies on Dip-Soldering Process of Printed Wiring Boards*
}

\author{
Shin-ichi KoyAnAGI**
}

\section{Introduction}

In the previous report, the various factors were explained which determined the reliability of electrical insulation on printed boards used to the permanental equipments such as carrier system, and in this paper the production control limit to prevent electrical failure of the parts and adhesion of conductors to the boards by heating during dip-soldering operation are reported.

\section{Conception about Dip-Soldering Operation}

Some merits of "Printed Circuit Method" are the saving of labour, the increase of soldering strength by the dip-soldering operation, and the possibility to secure accomplish soldering of many electrical components to the boards by one operation.

But on the other hand, when the boards are dipped into the molten solder, characteristic failure of the component and deterioration of adhesion of conductor due to heating are naturally inevitable, and so control limits about design and production must be determined.

\section{Experimentals}

As to following items investigation was made about dip-soldering processes.

a ) With the failure of component by heating during the process the author measured the change of characteristic styroflex capacitor, tantalum electrolytic capacitor and of germanium diode caused by dip-soldering.

From these results, within standard producing conditions, some changes in characteristics were noticed, but were not critical for practical uses.

b) About the pealing of copper foils, the author determined the strength relations between the diameter of component leads and direction of stresses, and from these results, the explanation could be made on following rules. The copper conductor foils are unable to withstand to heavy stresse applied by component leads if the applied stresses tend to pull the conductor foils away from the boards, but they are amply withstanding to stress directed toward the boards.

c) In Fig. 1 the deterioration of adhesive strength of conductor is shown when dip-soldering is repeated.

This result of deterioration suggests that repeated heating of printed boards is harmful, so some limitation of heating schedule of printed boards must be settled to maintiain the reliability for long use of the equipments.

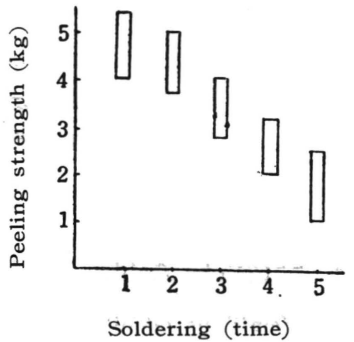

Fig. 1 Peeling strength u.s. soldering times of boars.

(Received October 14, 1958)

\section{Literature :}

(1) S. Koyanagi, J. Electrochem. Soc. Japan, 25, (1957)

(2) S. Koyanagi, J. Electrochem. Soc. Japan, 26, (1958)

(3) W. Hannahs et at, Tele-Tech, Feb. (1954)

* Studies on Printed Circuits (Part 3)

** Tamagawa Plant, Nippon Electric Co., Ltd. Kawasaki, Japan The original written in Japanese can be seen in J. Electrochem. Soc. Japan 27, 635 (1959). 